\section{Case Reports in Ophthalmology}

\section{Case Rep Ophthalmol 2013;4:114-121}

This is an Open Access article licensed under the terms of the Creative Commons Attribution-NonCommercial 3.0 Unported license (CC BY-NC) (www.karger.com/OAlicense), applicable to the online version of the article only. Distribution permitted for noncommercial purposes only.

\title{
Pathological Findings of Pemphigus Vulgaris Showing Giant Cobblestone-Like Conjunctival Papillae
}

\author{
Hiroyuki Namba ${ }^{a} \quad$ Mari Narumi $^{a} \quad$ Akira Sugano $^{a} \quad$ Ichidai Murata $^{b}$ \\ Tamio Suzuki $^{\mathrm{b}}$ Mitsunori Yamakawa ${ }^{c}$ Hidetoshi Yamashita $^{\mathrm{a}}$ \\ Departments of ${ }^{a}$ Ophthalmology and Visual Science, ${ }^{b}$ Dermatology and ${ }^{c}$ Diagnostic \\ Pathology, Yamagata University Faculty of Medicine, Yamagata City, Japan
}

\section{Key Words}

Conjunctiva $\cdot$ Ocular surface $\cdot$ Pathology $\cdot$ Immunology

\begin{abstract}
Purpose: To describe the pathophysiological findings of a patient with pemphigus vulgaris (PV) showing giant conjunctival papillae. Methods: A 64-year-old man who had mucosaldominant PV with giant conjunctival papillae, resembling those of vernal keratoconjunctivitis (VKC), underwent an ophthalmological workup. The clinical and pathological findings were investigated. Results: Ophthalmic interventions were unable to provide the desired beneficial effects, and multiple excisions were necessary to remove the proliferative conjunctival lesions. Histopathological investigations of the excised tissues demonstrated acantholysis and a subconjunctival infiltration with numerous inflammatory cells such as lymphocytes, plasma cells, and neutrophils. However, in contrast to typical VKC, mast cells and eosinophils were rarely found in the subconjunctival tissues. Direct immunofluorescent staining showed a significant deposition of immunoglobulin $\mathrm{G}$ and complement component 3 in the epithelial intercellular substance, consistent with mucosal-dominant PV. Then, the patient was hospitalized because of oral erosion exacerbation and malnutrition. Because of the patient's declining general condition, we administered an increasing dose of a systemic steroid with an intravenous immunoglobulin, after which his ocular lesions and symptoms improved. Conclusions: The histological conjunctival papilla findings were quite different from those of VKC papillae. If PV causes a lesion in a patient, systemic immunosuppression might be more effective than topical ophthalmic treatment because of overall immunological involvement.
\end{abstract}


Namba et al.: Pathological Findings of Pemphigus Vulgaris Showing Giant Cobblestone-Like Conjunctival Papillae

\section{Introduction}

Pemphigus is an autoimmune, intraepithelial blistering disease affecting the skin and mucosa. The condition is characterized by a loss of adhesion between epithelial cells, resulting from acantholysis and epithelial and blood serum autoantibody presence [1]. These autoantibodies target desmoglein 1 (Dsg1) and desmoglein 3 (Dsg3), which play important roles in epithelial cell-cell adhesion at cellular junctures known as desmosomes.

Pemphigus can be divided into 3 main types: pemphigus vulgaris (PV), pemphigus foliaceus, and paraneoplastic pemphigus (PNP) [2]. PV is the most frequent type, representing about $70 \%$ of pemphigus cases; it is characterized clinically by blisters and erosions both in the mucous membranes and on the skin. It is classified as mucosal-dominant and mucocutaneous. The former has only anti-Dsg3 antibodies, whereas the latter has both anti-Dsg1 and anti-Dsg3 antibodies [1]. Pemphigus foliaceus is characterized clinically by whole-body skin blisters and erosions; however, blisters are not seen in the mucous membranes, as only the anti-Dsg1 antibodies are present [2]. Meanwhile, PNP develops in association with malignancies such as non-Hodgkin's lymphoma, chronic lymphocytic leukemia, Castleman's disease, and thymoma [3]. It is characterized by oral mucosal erosions and multiforme skin eruptions consisting of whole-body skin erythemas, blisters, and erosions [1].

Reports of ocular involvement with pemphigus are rare, and the majority of cases involve cicatricial conjunctivitis [4-6]. Proliferative palpebral conjunctival lesions are rarely investigated; thus, reports evaluating the pathological findings are important because they could enable a better understanding of the pathogenesis of ocular involvement.

Giant cobblestone-like papillae are proliferative changes in the palpebral conjunctiva and usually develop in vernal keratoconjunctivitis (VKC). VKC is a chronic, bilateral allergic inflammation involving the tarsal conjunctiva, bulbar conjunctiva, or both, and often leads to cornea ulceration, including pruritus, pain, hyperemia, and photophobia [7]. Further, VKC has a complex pathophysiology resulting from relationships among mast cells, helper T cells, eosinophils, epithelial cells, fibroblasts, etc. [8].

Herein, we present a patient with PV who had conjunctival giant papillae with symptoms observed in VKC and report the patient's clinical, histopathological, and immunopathological findings.

\section{Case Report}

This study was performed in accordance with the Declaration of Helsinki, and approval was obtained from the ethics committee at the Yamagata University Faculty of Medicine. Our patient was a 64-year-old man who had mucosal-dominant PV with giant conjunctival papillae. His clinical course and histopathological and immunopathological findings were investigated.

Since 2007, the patient's oral mucosal erosions had been treated at another hospital. In a direct immunofluorescence assay, an oral mucosal biopsy showed acantholysis, immunoglobulin G (IgG), and complement component 3 (C3) deposits around the epithelial cells. In addition, a blood serum examination showed the presence of anti-Dsg3 autoantibodies. On the basis of these results, he was diagnosed with mucosal-dominant PV. He was treated with prednisolone, cyclosporine A, and mizoribine. However, the PV appeared to be insufficiently controlled as the patient had repeated remissions and deteriorations. In 2010, proliferative papillary lesions appeared on the bilateral palpebral conjunctivas. The ocular lesions were treated at the other ophthalmic clinic with topical steroids and cyclosporine A. However, the 
Namba et al.: Pathological Findings of Pemphigus Vulgaris Showing Giant Cobblestone-Like Conjunctival Papillae

management was unsuccessful, as evidenced by marked papilla proliferation and elevated intraocular pressure (IOP). Hence, the giant papillae were excised several times. In March 2011, after moving to our town, he was admitted to our hospital, where the Departments of Dermatology and Ophthalmology attempted to control the PV and the proliferative papillary lesions, respectively.

At the time of his first admission to our ophthalmic clinic, he had pruritus, pain, and photophobia. An ophthalmic examination showed a corrected visual acuity of 20/20 in the right eye and 20/25 in the left eye and an IOP of 15 and $18 \mathrm{~mm} \mathrm{Hg}$ in the right eye and left eye, respectively. Both the eyelids were edematous, and the superior palpebral conjunctivas showed giant cobblestone-like papillae mostly laterally (fig. 1a, b). Both corneas were clear, with deep and quiet anterior chambers. Both eyes were phakic, and the posterior segments were unremarkable. The patient also experienced throat soreness and odynophagia. Otolaryngological examinations revealed mucosal erosions on the tongue, pharynx, and esophagus. However, as the condition lacked cutaneous involvement, it was inferred to be mucosal-dominant PV. The anti-Dsg3 antibody index value was greater than 150 . The dermatologist subsequently administered a daily dose of $15 \mathrm{mg}$ oral prednisolone. We started ophthalmic treatment with a $0.1 \%$ topical tacrolimus solution 4 times daily, and $0.25 \%$ prednisolone ointment once daily. As the efficacy was insufficient, instead of tacrolimus, topical steroids $(0.1 \%$ fluorometholone or $0.1 \%$ betamethasone) were applied. However, the IOP was mostly elevated; thus, the topical steroid treatments were deemed to be insufficiently intensified. In July, the giant papillae were surgically excised. Nonetheless, subsequent symptom control, including palpebral conjunctival findings, remained inadequate with topical administration. Therefore, additional surgical excisions were performed in August and October, after which the excised tissues were investigated pathologically.

Hematoxylin and eosin (HE) staining demonstrated prominent inflammatory cells including lymphocytes, plasma cells, and neutrophils, but infrequent mast cells and eosinophils in the subconjunctival tissue. Marked acantholysis was observed in the epithelium (fig. 1d). Direct immunofluorescent staining showed a significant deposition of IgG and C3 in the epithelial intercellular spaces (fig. 1d, f). Toluidine blue staining and c-kit immunostaining showed minimal mast cells (fig. 2c, d). Immunostaining also showed $\mathrm{CD}^{+}{ }^{+} \mathrm{T}$ cells had infiltrated to almost the same degree as $\mathrm{CD}^{+}$cells (fig. 2e, f). Immunostaining of CD20 and CD79a indicated numerous mature B cell and plasma cell infiltrations (fig. 2g, h).

In early November, the patient was hospitalized at the Department of Dermatology for difficulty in eating and oral erosion exacerbation. The anti-Dsg3 antibody index value was greater than 150, and the anti-Dsg1 index value was elevated at 50. Although the patient began treatment with a 3-mg/kg dose of cyclosporine A daily and a 15-mg dose of oral prednisolone daily, he did not have complete resolution. Therefore, prednisolone was increased to $60 \mathrm{mg}$ daily in late November. However, the treatment was unsatisfactory; therefore, in December, prednisolone was increased to $90 \mathrm{mg}$ daily with a $400-\mathrm{mg} / \mathrm{kg} / \mathrm{day}$ intravenous immunoglobulin dose for 5 days; cyclosporine A was simultaneously ceased. Intravenous immunoglobulin treatment was selected for its immunological superiority, as compared to steroid pulse or plasmapheresis. The anti-Dsg1 and anti-Dsg3 antibody index values gradually decreased and were accompanied by oral erosion improvement and the ability to eat (fig. 3). The ophthalmic symptoms and conjunctival giant papillae resolved with the increased systemic treatment (fig. 1c). These improvements occurred more quickly than those of the oral lesions. The IOP did not increase during treatment. 
Namba et al.: Pathological Findings of Pemphigus Vulgaris Showing Giant Cobblestone-Like Conjunctival Papillae

\section{Discussion}

To our knowledge, this is the first report of PV showing conjunctival giant papillae. As ocular involvement in PV is rare, histopathological investigations of periocular lesions have seldom been examined. Importantly, this report considers the pathogenesis of ocular involvement.

Palpebral giant cobblestone-like papillae are frequently observed in VKC; however, the histopathological appearance of our patient's papillae was quite different from that of VKC papillae. In particular, despite significant inflammatory cell infiltration, mast cells and eosinophils were almost absent. An allergic disease course can typically be divided into 2 phases: immediate hypersensitivity reaction (early- or acute-phase reaction) and late-phase reaction. Mast cell activation plays an important role in the immediate hypersensitivity reaction, and eosinophils are principal effecters in the late-phase reaction [9]. The lack of mast cell and eosinophil infiltrations suggests that an allergic mechanism did not occur on the conjunctiva; moreover, the ocular lesions may not have resulted from an allergic disease.

One relevant question is whether the ocular lesions were truly generated by PV. The patient was diagnosed with mucosal-dominant PV based on clinical appearance. In addition to anti-Dsg3 antibodies, anti-Dsg1 antibodies were detected throughout treatment. Notably, previous reports have shown that some PV patients develop anti-Dsg1 antibodies during the disease course $[10,11]$. Based on the above-mentioned consideration, another relevant issue was whether PNP was present. In PNP, inflammatory changes with subepithelial lymphocytic infiltrations are also observed. Moreover, PNP is characterized by both anti-Dsg1 and antiDsg3 antibodies. These features are not contradictory to the findings in the case. Nevertheless, no signs of malignancy were observed in the blood or computed tomography examinations during hospitalization. Subsequently, type 0 -IIc early gastric cancer was detected by an endoscopic exam in 2012. However, it seems unlikely that the early cancer was present in 2007, and PNP has never been reported in gastric cancer. Additionally, our patient had conjunctival giant papilla symptoms again with systemic steroid reduction, despite the complete excision of the cancer. Previous reports showed that cases with benign resectable tumors may have clinical symptom improvement with tumor resection $[12,13]$.

Pemphigus is a systemic autoimmune disease, possibly explaining why our topical treatments had no effect. Autoimmune reactions were probably activated in the other systemic epithelial lesions despite topical ophthalmic treatment. If so, systemic immunosuppression may be more effective than local treatment, although ophthalmologists tend to treat with topical solutions. Moreover, the patient was considered a steroid responder. Nonetheless, despite the increased systemic steroid dosage, the IOP decreased. A previous report demonstrated a higher frequency of IOP elevation with topical steroids compared to that of systemic forms, which might explain this outcome [14]. Further, it may have also resulted from the excision of the proliferative eyelid tissue that removed pressure on the eye.

Moreover, this case is atypical for both PV and PNP. Nevertheless, ophthalmologists should consider that pemphigus sometimes has ophthalmological involvement, including the rare occurrence of giant conjunctival papillae.

\section{Acknowledgements}

The authors would like to thank Kentaro Kobayashi for clinical data collection and Tomoya Kato and Hiroya Otake for their technical skills. 


\begin{tabular}{l|l}
\hline Case Rep Ophthalmol 2013;4:114-121 \\
\hline DOI: $10.1159 / 000355374$ & $\begin{array}{l}\text { C 2013 S. Karger AG, Basel } \\
\text { www.karger.com/cop }\end{array}$ \\
\hline
\end{tabular}

Namba et al.: Pathological Findings of Pemphigus Vulgaris Showing Giant Cobblestone-Like Conjunctival Papillae

\section{Disclosure Statement}

The authors received no specific grants from any funding agency in the public, commercial, or not-for-profit sectors. No conflicting relationships exist for any author.

\section{References}

1 Kitajima Y, Aoyama Y: A perspective of pemphigus from bedside and laboratory-bench. Clin Rev Allergy Immunol 2007;33:57-66.

-2 Joly P, Litrowski N: Pemphigus group (vulgaris, vegetans, foliaceus, herpetiformis, brasiliensis). Clin Dermatol 2011;29:432-436.

-3 Wade MS, Black MM: Paraneoplastic pemphigus: a brief update. Australas J Dermatol 2005;46:1-8.

-4 Daoud YJ, Cervantes R, Foster CS, Ahmed AR: Ocular pemphigus. J Am Acad Dermatol 2005;53:585-590.

5 Olszewska M, Komor M, Mazur M, Rogozinski T: Response of ocular pemphigus vulgaris to therapy. Case report and review of literature. J Dermatol Case Rep 2008;2:1-3.

6 Tam PM, Cheng LL, Young AL, Lam PT: Paraneoplastic pemphigus: an uncommon cause of chronic cicatrising conjunctivitis. BMJ Case Rep 2009;2009:bcr12.2008.1306.

7 Kumar S: Vernal keratoconjunctivitis: a major review. Acta Ophthalmol 2009;87:133-147.

-8 Kumagai N, Fukuda K, Fujitsu Y, Yamamoto K, Nishida T: Role of structural cells of the cornea and conjunctiva in the pathogenesis of vernal keratoconjunctivitis. Prog Retin Eye Res 2006;25:165-187.

-9 Miyazaki D, Nakamura T, Toda M, Cheung-Chau KW, Richardson RM, Ono SJ: Macrophage inflammatory protein-1alpha as a costimulatory signal for mast cell-mediated immediate hypersensitivity reactions. J Clin Invest 2005; 115:434-442.

10 Harman KE, Gratian MJ, Bhogal BS, Challacombe SJ, Black MM: A study of desmoglein 1 autoantibodies in pemphigus vulgaris: racial differences in frequency and the association with a more severe phenotype. $\mathrm{Br} J$ Dermatol 2000;143:343-348.

-11 Mortazavi H, Shahdi M, Amirzargar AA, Naraghi ZS, Valikhani M, Daneshpazhooh M, Vasheghani-Farahani A, Sedaghat M, Chams-Davatchi C: Desmoglein ELISA in the diagnosis of pemphigus and its correlation with the severity of pemphigus vulgaris. Iran J Allergy Asthma Immunol 2009;8:53-56.

12 Menenakos C, Braumann C, Hartmann J, Jacobi CA: Retroperitoneal Castleman's tumor and paraneoplastic pemphigus: report of a case and review of the literature. World J Surg Oncol 2007;5:45.

13 Barbetakis N, Samanidis G, Paliouras D, Boukovinas I, Asteriou C, Stergiou E, Laschos K, Tsilikas C: Paraneoplastic pemphigus regression after thymoma resection. World J Surg Oncol 2008;6:83.

14 Schwartz B: The response of ocular pressure to corticosteroids. Int Ophthalmol Clin 1966;6:929-989. 
Namba et al.: Pathological Findings of Pemphigus Vulgaris Showing Giant Cobblestone-Like Conjunctival Papillae
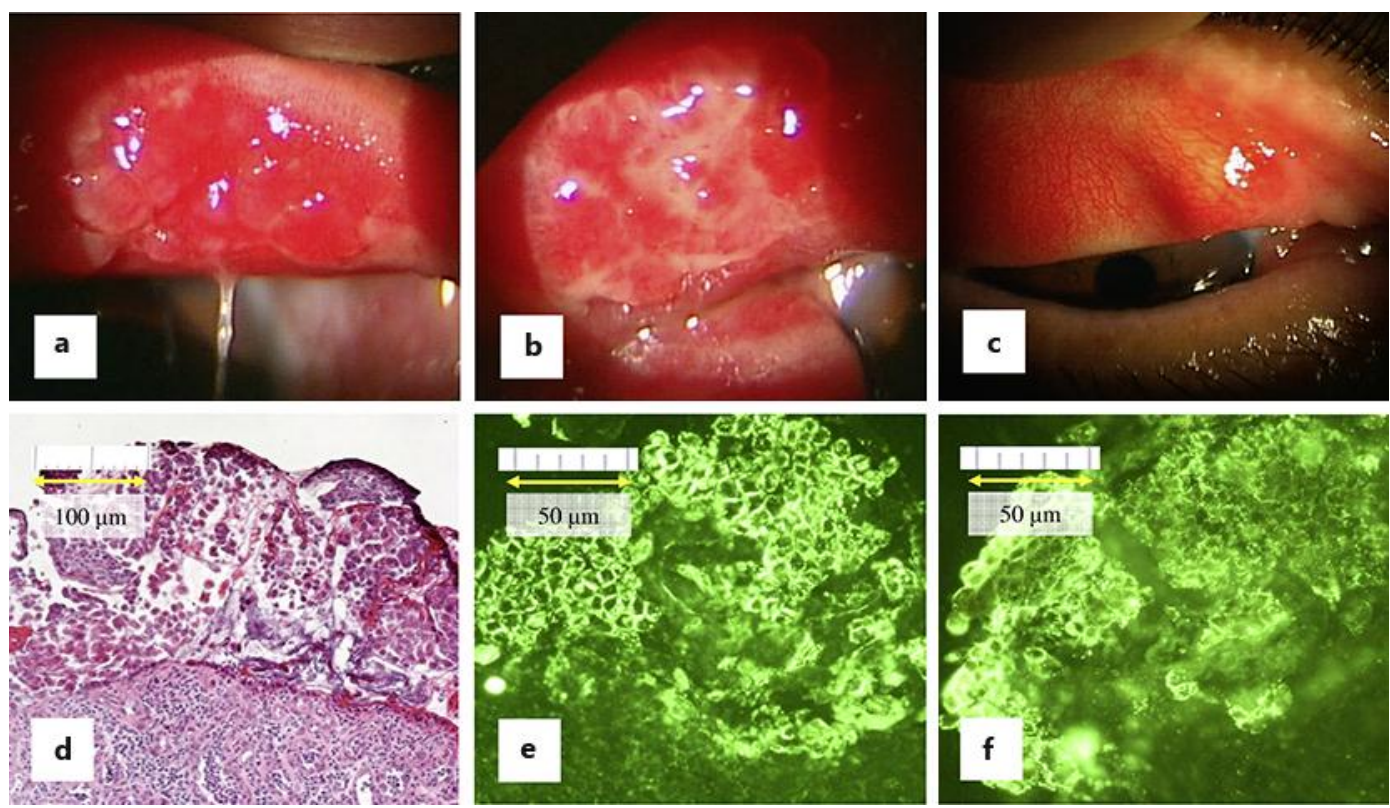

Fig. 1. Slit lamp photograph, histology, and direct immunofluorescent staining of palpebral conjunctiva. a Right conjunctiva. b Left conjunctiva. c Left conjunctiva in December 2010. The giant papillae resolved with increased systemic treatment. $\mathbf{d}$ Resected tissues revealed marked inflammatory reaction. HE. Note epithelial acantholysis and marked inflammatory cell infiltration, including lymphocytes, plasma cells, and neutrophils in the subconjunctival tissue. e, f Direct immunofluorescent staining of IgG (e) and C3 (f) showing the intercellular deposition of each protein in the epithelium. 


\section{Case Reports in \\ Ophthalmology}

\begin{tabular}{l|l}
\hline Case Rep Ophthalmol 2013;4:114-121 \\
\hline DOI: 10.1159/000355374 & $\begin{array}{l}\text { ○ 2013 S. Karger AG, Basel } \\
\text { www.karger.com/cop }\end{array}$ \\
\hline
\end{tabular}

Namba et al.: Pathological Findings of Pemphigus Vulgaris Showing Giant Cobblestone-Like Conjunctival Papillae
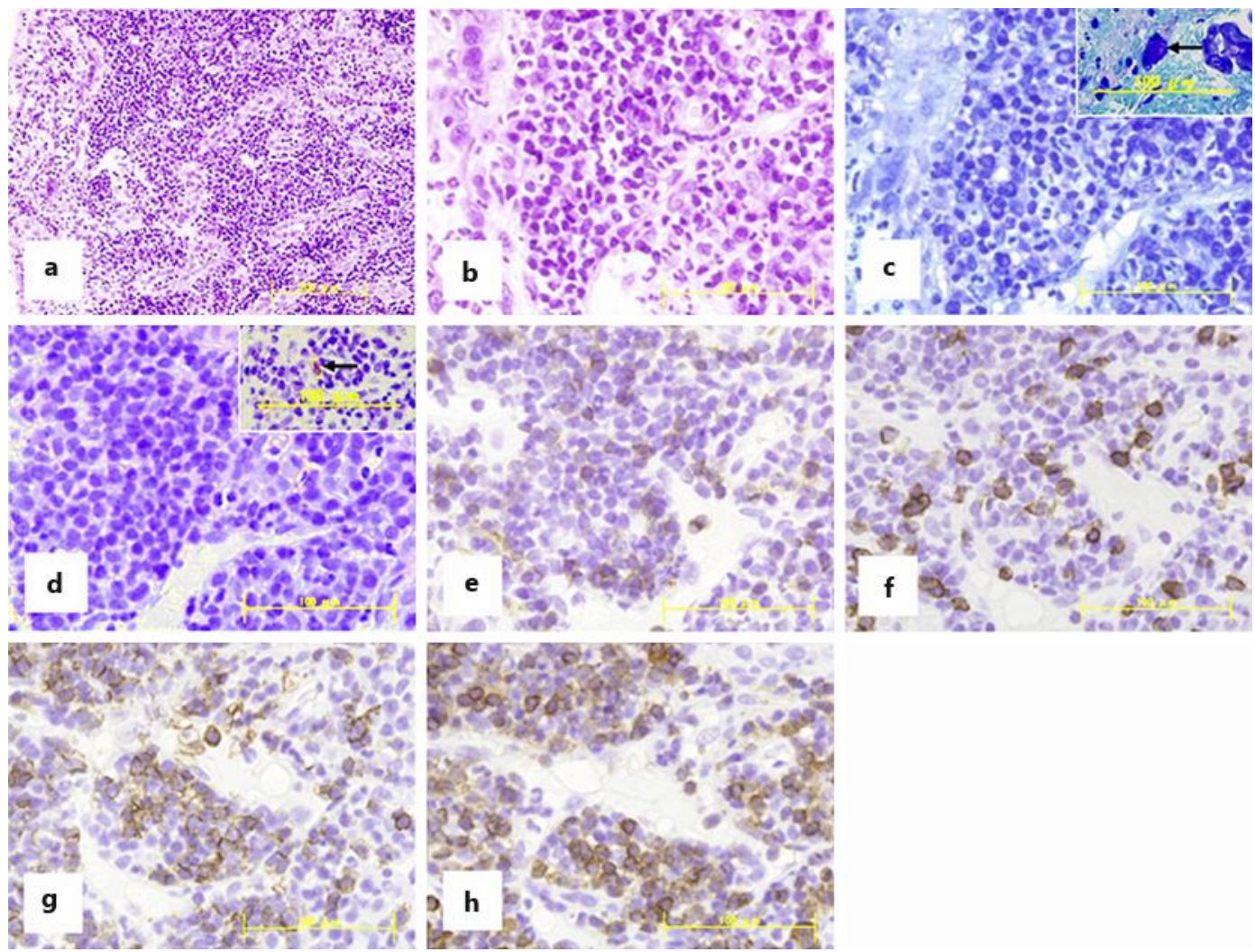

Fig. 2. Histology and immunohistochemical staining in the subconjunctival tissue. a Note marked inflammatory cell infiltration. HE. b Inflammatory cells composed of lymphocytes, plasma cells, and a few neutrophils. HE. c Toluidine blue staining revealed that the subconjunctival tissue infrequently contained mast cells. The inset shows a mast cell with metachromasia (arrow). d C-kit immunostaining reveals only a few mast cells. The inset shows a positive cell (arrow). Immunohistochemical findings of CD4 (e), CD8 (f), CD20 (g), and CD79a (h) in the resected subconjunctival tissue. CD4+ T cells are represented almost equally with $\mathrm{CD}^{+} \mathrm{T}$ cells, and $\mathrm{CD}^{+} 9 \mathrm{a}^{+}$cells predominate over $\mathrm{CD} 20^{+}$cells, indicating plasma cell infiltration. 
Namba et al.: Pathological Findings of Pemphigus Vulgaris Showing Giant Cobblestone-Like Conjunctival Papillae

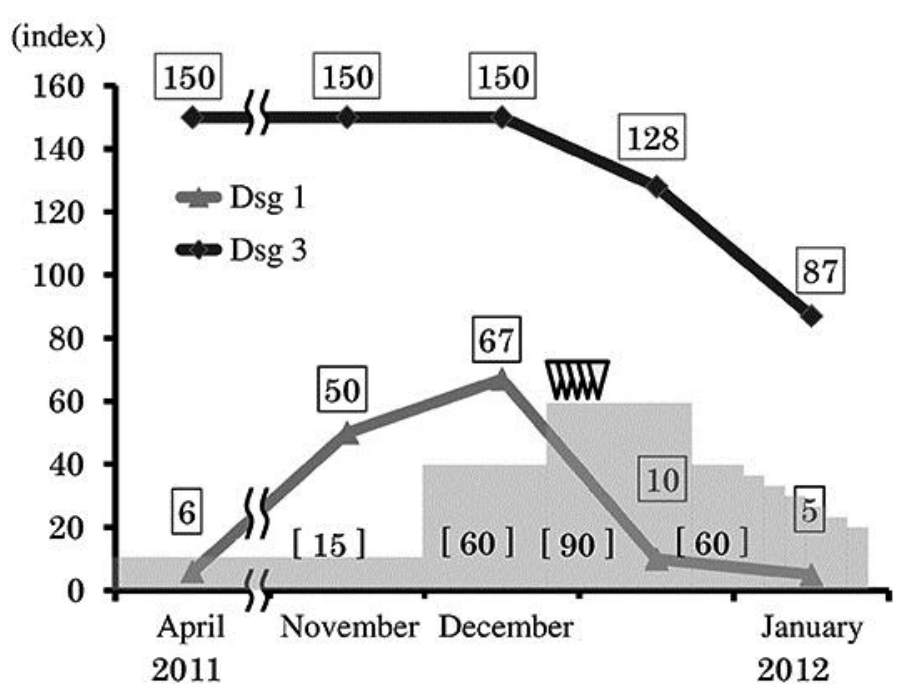

Fig. 3. Clinical course and index value transitions. Anti-Dsg1 and anti-Dsg3 antibody index values gradually decreased with increased systemic treatment. Dsg1 = Anti-Dsg1 antibody index (values shown in squares); Dsg3 = anti-Dsg3 antibody index (values shown in squares); light grey meshing = prednisolone application (doses shown in brackets; $\mathrm{mg} /$ day); arrowheads = intravenous immunoglobulin administration. 